\title{
Unravelling mechanisms behind biomass-clay interactions using comprehensive multiphase nuclear magnetic resonance (NMR) spectroscopy
}

Melisa S. Olivelli†¥, Ioana Fugariu¥, Rosa M. Torres Sánchez $¥$, Gustavo Curutchet†¥, André J. Simpson $¥ *$ and Myrna J. Simpson $¥ *$.

†Instituto de Investigación e Ingeniería Ambiental-IIIA, UNSAM, CONICET, 3iA, Campus Miguelete, 25 de mayo y Francia, 1650-San Martín, Provincia de Buenos Aires, Argentina.

‡Environmental NMR Centre and Department of Physical and Environmental Sciences, University of Toronto Scarborough, 1265 Military Trail, Toronto, ON, Canada M1C 1A4

YCentro de Tecnología de Recursos Minerales y Cerámica (CETMIC). CIC-CCT-La Plata. Camino Centenario y 506, M.B. Gonnet, Buenos Aires, Argentina.

¥Consejo nacional de investigaciones científicas y técnicas - CONICET. Buenos Aires, Argentina.

*Corresponding authors:

André J. Simpson. E-mail: andre.simpson@utoronto.ca

Myrna J. Simpson. E-mail: myrna.simpson@utoronto.ca 

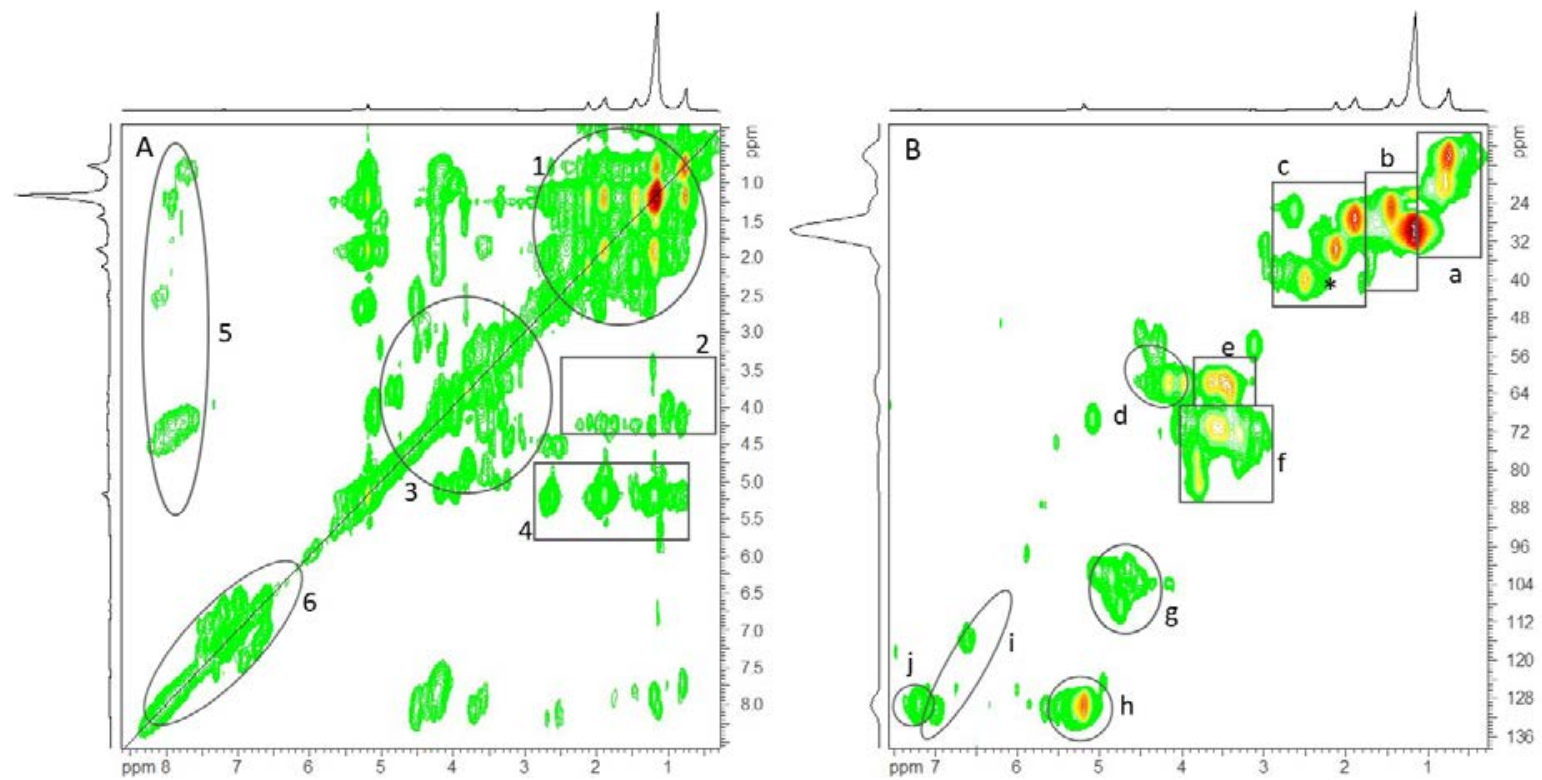

Figure S1. (A): ${ }^{1} \mathrm{H}-{ }^{1} \mathrm{H}$ TOCSY NMR spectrum at $500 \mathrm{MHz}$ of Apha biomass: (1) Aliphatic chains; (2) correlation between $\alpha-\mathrm{H}$ and peptide side chains in amino acids, peptides and proteins; (3) signals from carbohydrates; (4) correlation of unsaturation to aliphatic chains from lipids; (5) correlation between amides to $\alpha$-H and side chains from proteins/peptides; (6) signals from aromatic compounds. (B): ${ }^{1} \mathrm{H}-{ }^{13} \mathrm{C}$ HSQC NMR spectrum of Apha biomass: (a) $\mathrm{CH}_{3}$ from lipids and/or peptides; (b) $\mathrm{CH}_{2}$ in lipids; (c) $\mathrm{C}$-H bonds in various aliphatic structures including fatty acids and amino acids; (d) $\alpha-\mathrm{H} / \mathrm{C}$ in peptides; (e) $\mathrm{CH}_{2}$ in carbohydrates; (f) C-H bonds in carbohydrates; (g) anomeric $\mathrm{H}$ (carbohydrates); and (h) $\mathrm{C}=\mathrm{C}$ from double bonds in aliphatic chains; (i) Tyrosine; (j) Phenylalanine. *DMSO= dimethyl sulfoxide. 

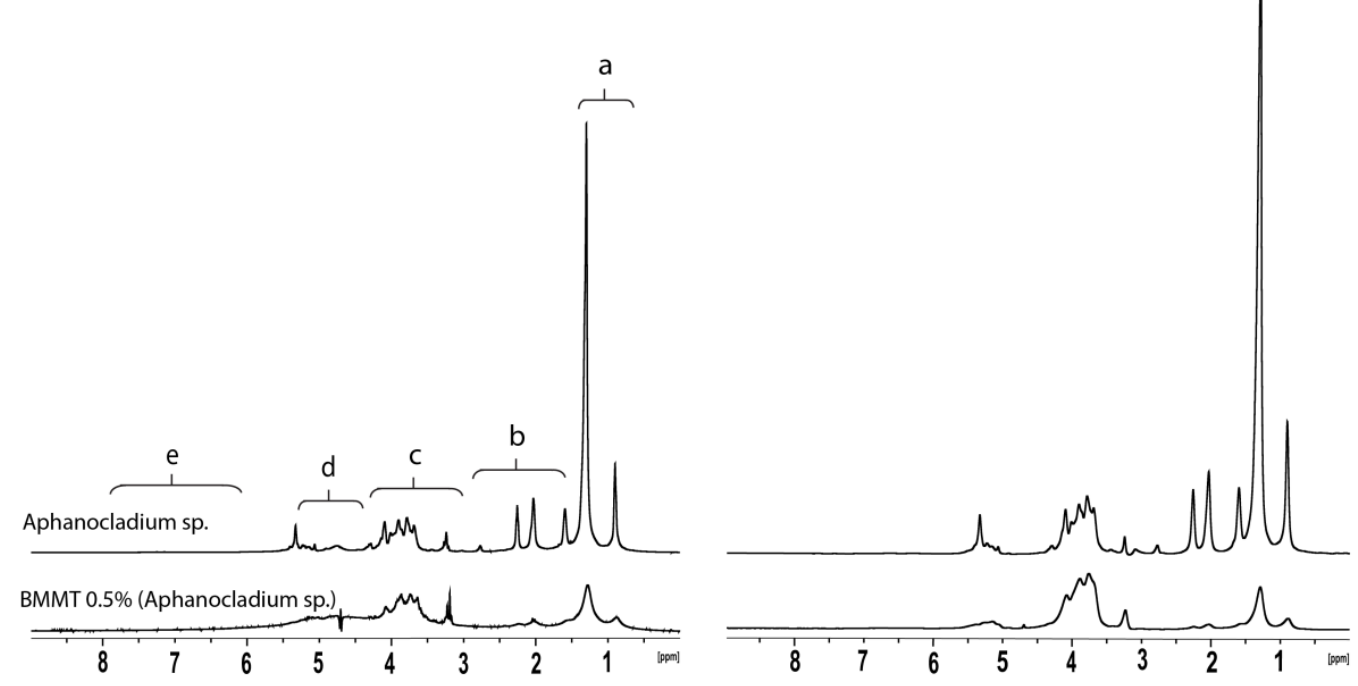

Figure S2. (A), ${ }^{1} \mathrm{H}$ CMP-MAS NMR spectra of BMMT (Apha) at different loading of biomass in $\mathrm{D}_{2} \mathrm{O}$. (B), ${ }^{1} \mathrm{H}$ CMP-MAS NMR spectra of BMMT (Apha) at different loading of biomass in $\mathrm{D}_{2} \mathrm{O}$ using a diffusion edited experiment. Assignments are as follows: (a) terminal methyl and methylene groups between 0.6-1.3 ppm; (b) signals due to $O$ - and $\mathrm{N}$ substituted aliphatic structures in the 1.3-2.9 ppm region; (c) signals due to $O$-alkyl structures mainly from carbohydrates in the 2.9-4.1 ppm region; (d) overlapping weak resonances in the 4.1-5.4 ppm region generally attributed to a number of moieties including $\alpha-\mathrm{H}$ from peptides, anomeric $\mathrm{H}$ in carbohydrates and double bonds; and (e) signals from the aromatic structures, in the 6.2-7.8 ppm region. 


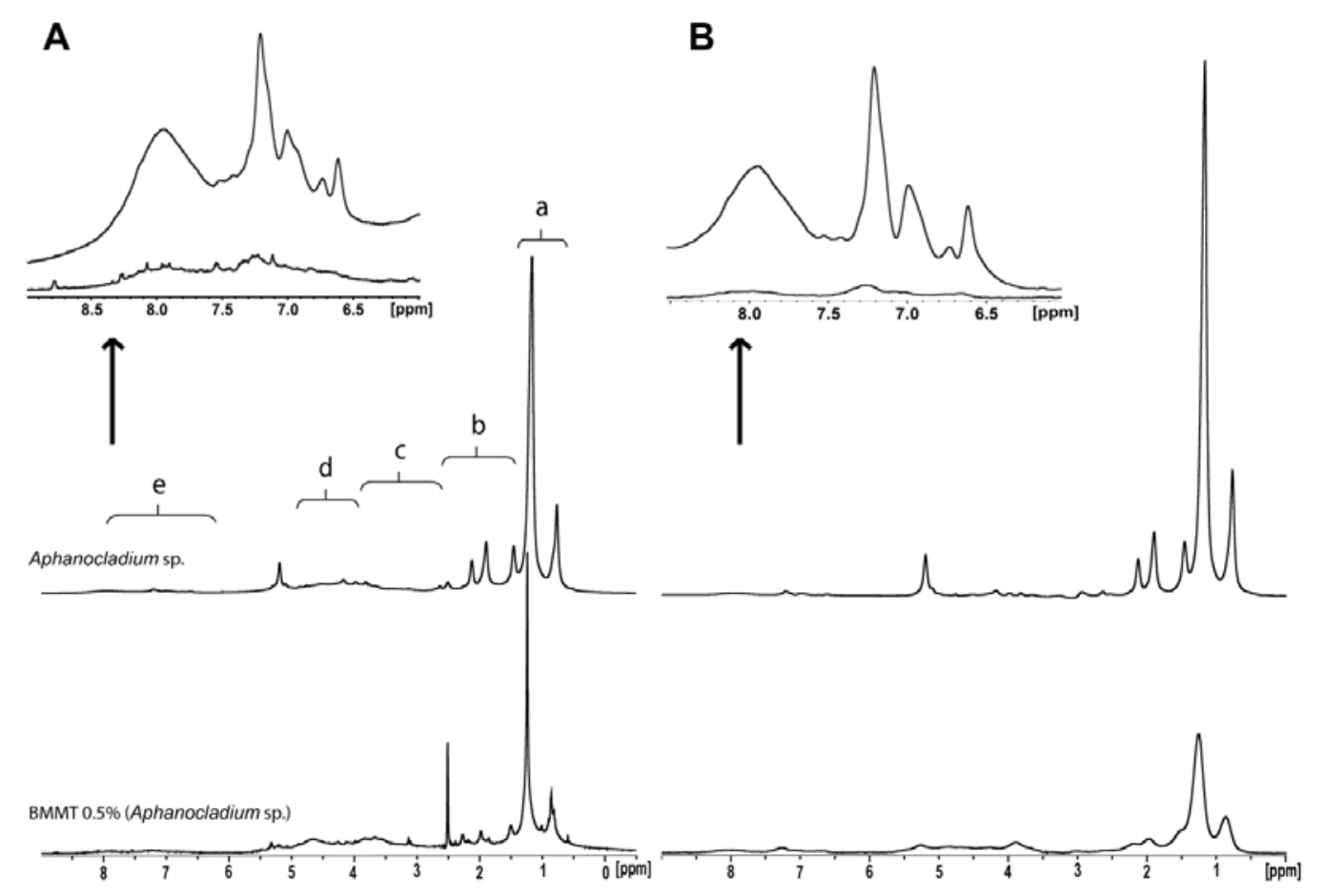

Figure S3. (A), ${ }^{1} \mathrm{H}$ CMP-MAS NMR spectra of BMMT (Apha) at different loading of biomass in DMSO-d $\mathrm{d}_{6}$. (B), ${ }^{1} \mathrm{H}$ CMP-MAS NMR spectra of BMMT (Apha) at different loading of biomass in DMSO- $\mathrm{d}_{6}$ using diffusion edited experiment. Arrow indicates a detail of the proteins region (9-6 ppm). Assignments are as follows: (a) terminal methyl and methylene groups between 0.6-1.3 ppm; (b) signals due to $O$ - and $N$ - substituted aliphatic structures in the 1.3-2.9 ppm region; (c) signals due to $O$-alkyl structures mainly from carbohydrates in the 2.9-4.1 ppm region; (d) overlapping weak resonances in the 4.1-5.4 ppm region generally attributed to a number of moieties including $\alpha-\mathrm{H}$ from peptides, anomeric $\mathrm{H}$ in carbohydrates and double bonds; and (e) signals from the aromatic structures, in the 6.2-7.8 ppm region. 
A

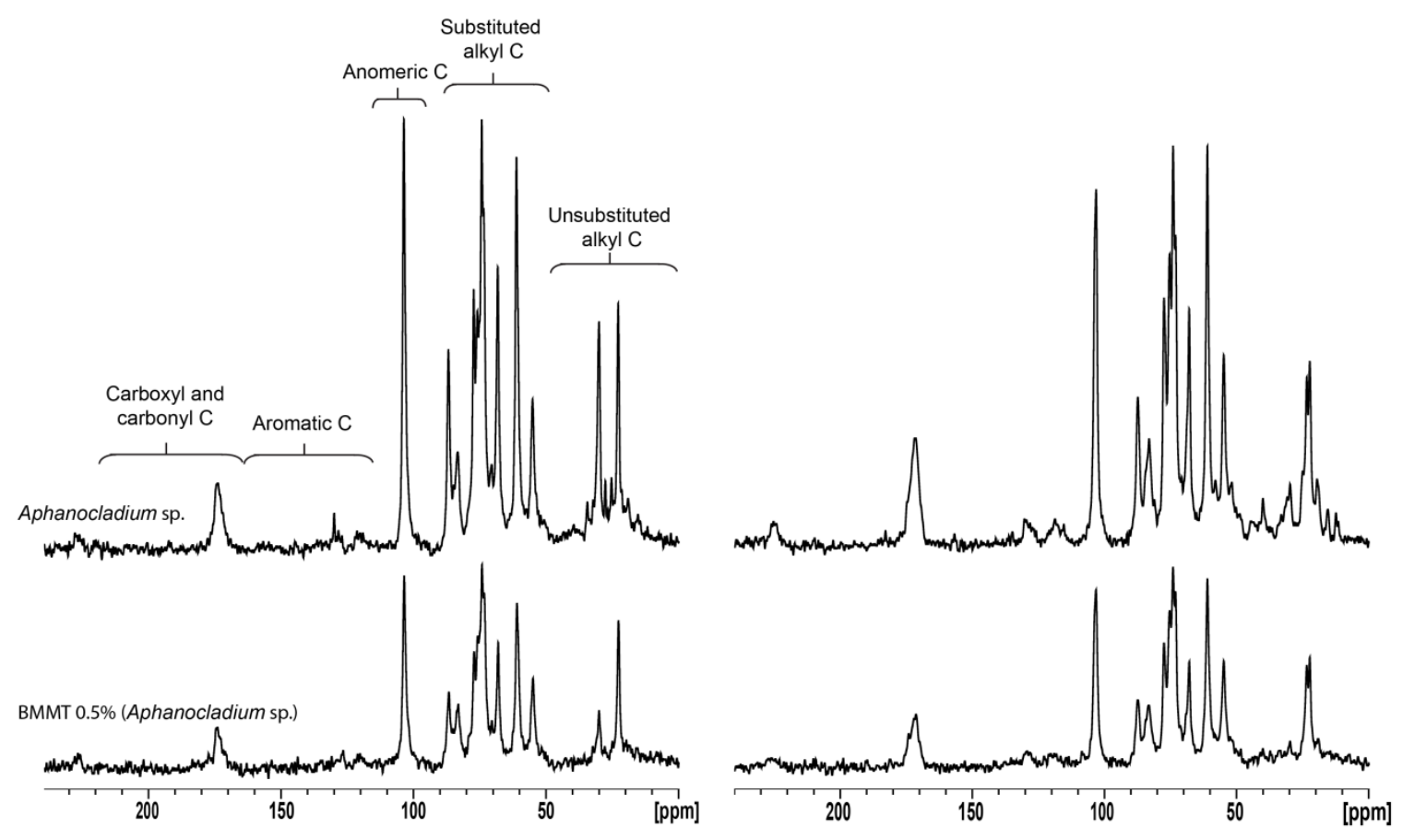

Figure S4. ${ }^{13} \mathrm{C}$ CP-MAS NMR spectra of BMMT (Apha) at different loading of biomass in (A), $\mathrm{D}_{2} \mathrm{O}$ and (B), DMSO-d 6 . 
A
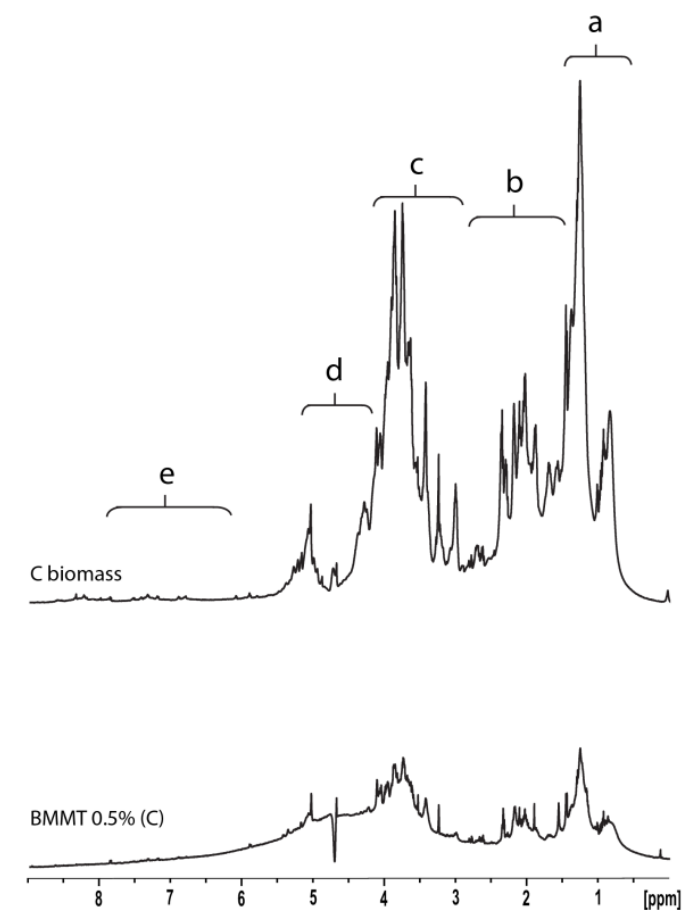

B

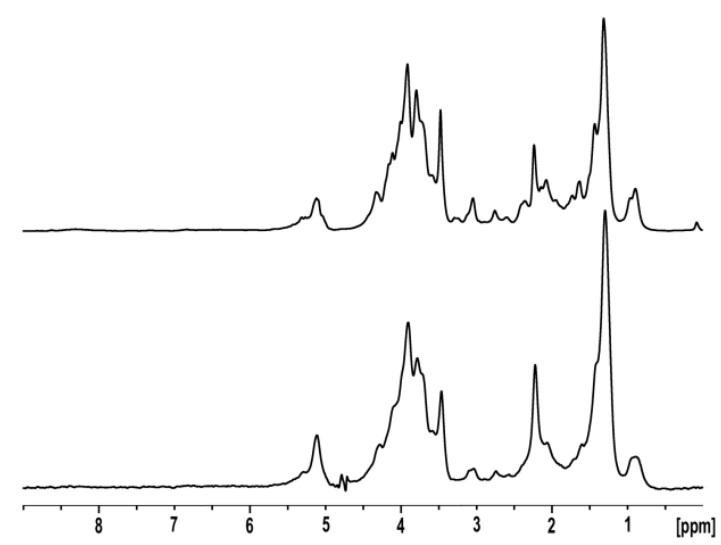

Figure S5. (A), ${ }^{1} \mathrm{H}$ CMP-MAS NMR spectra of BMMT (C) at different loading of biomass in $\mathrm{D}_{2} \mathrm{O}$. (B), ${ }^{1} \mathrm{H}$ CMP-MAS NMR spectra of BMMT (C) at different loading of biomass in $\mathrm{D}_{2} \mathrm{O}$ using a diffusion edited experiment. Assignments are as follows: (a) terminal methyl and methylene groups between 0.6-1.3 ppm; (b) signals due to $O$ - and $N$ - substituted aliphatic structures in the 1.3-2.9 ppm region; (c) signals due to $O$-alkyl structures mainly from carbohydrates in the 2.9-4.1 ppm region; (d) overlapping weak resonances in the 4.1$5.4 \mathrm{ppm}$ region generally attributed to a number of moieties including $\alpha-\mathrm{H}$ from peptides, anomeric $\mathrm{H}$ in carbohydrates and double bonds; and (e) signals from the aromatic structures, in the 6.2-7.8 ppm region. 

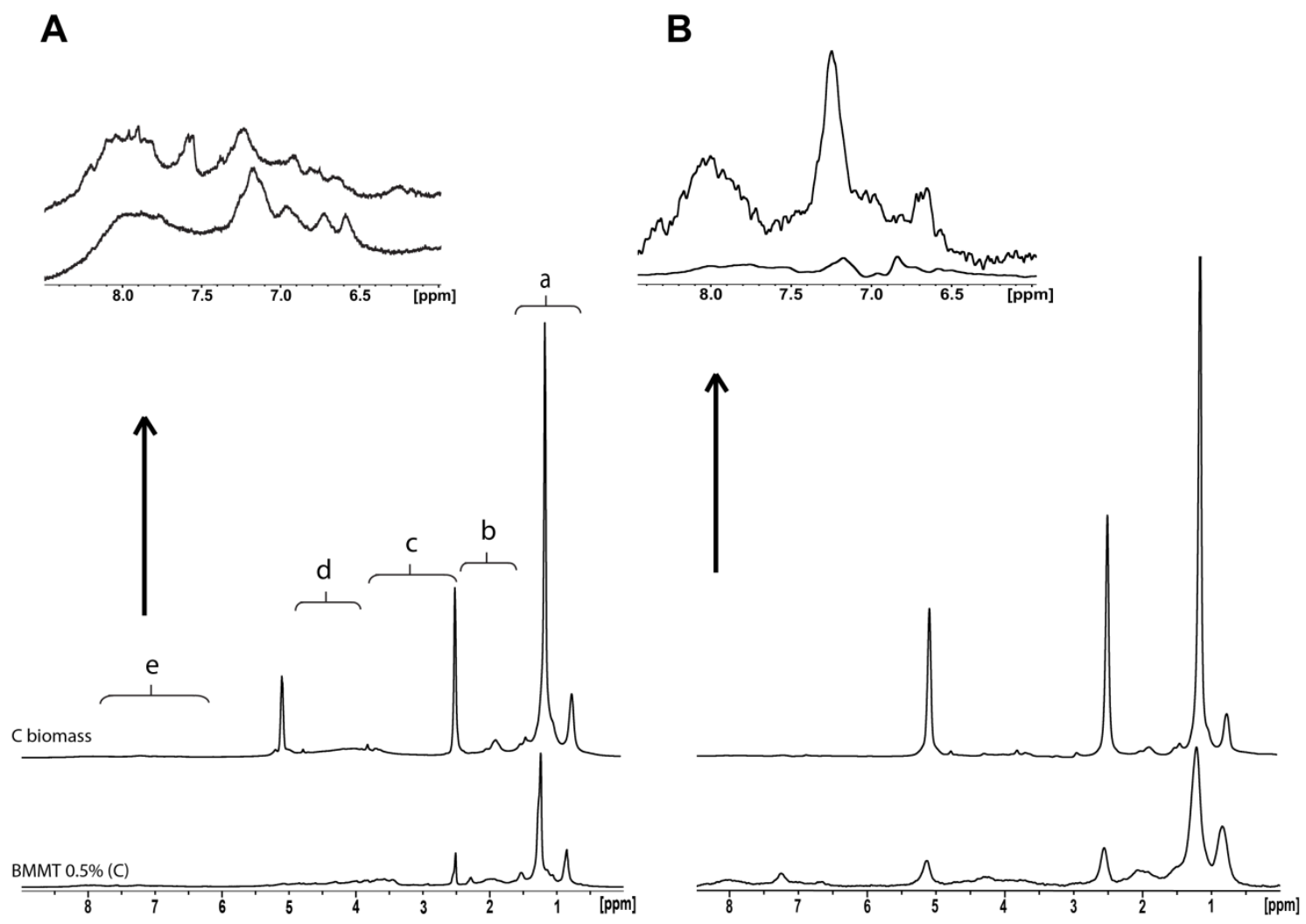

Figure S6. (A), ${ }^{1} \mathrm{H}$ CMP-MAS NMR spectra of BMMT (C) at different loading of biomass in DMSO- $\mathrm{d}_{6}$. (B), ${ }^{1} \mathrm{H}$ CMP-MAS NMR spectra of BMMT (C) at different loading of biomass in DMSO- $\mathrm{d}_{6}$ using diffusion edited experiment. The arrows indicate a detail of the proteins region (9-6 ppm). Assignments are as follows: (a) terminal methyl and methylene groups between 0.6-1.3 ppm; (b) signals due to $O$ - and $N$ - substituted aliphatic structures in the 1.3-2.9 ppm region; (c) signals due to $O$-alkyl structures mainly from carbohydrates in the 2.9-4.1 ppm region; (d) overlapping weak resonances in the 4.1-5.4 ppm region generally attributed to a number of moieties including $\alpha-\mathrm{H}$ from peptides, anomeric $\mathrm{H}$ in carbohydrates and double bonds; and (e) signals from the aromatic structures, in the 6.2-7.8 ppm region. 
A

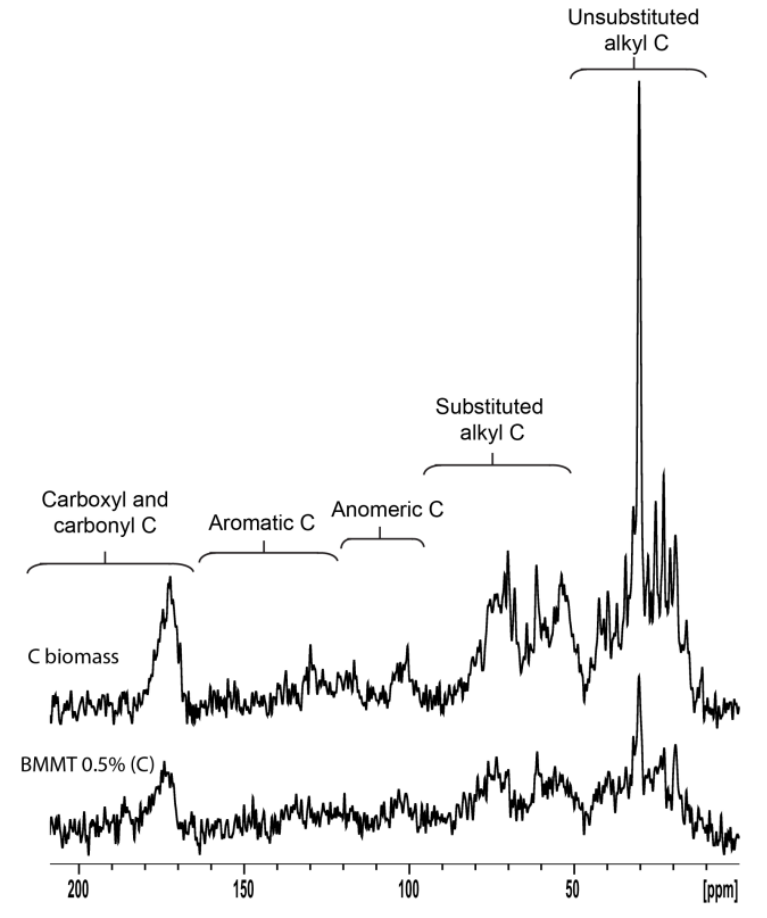

B

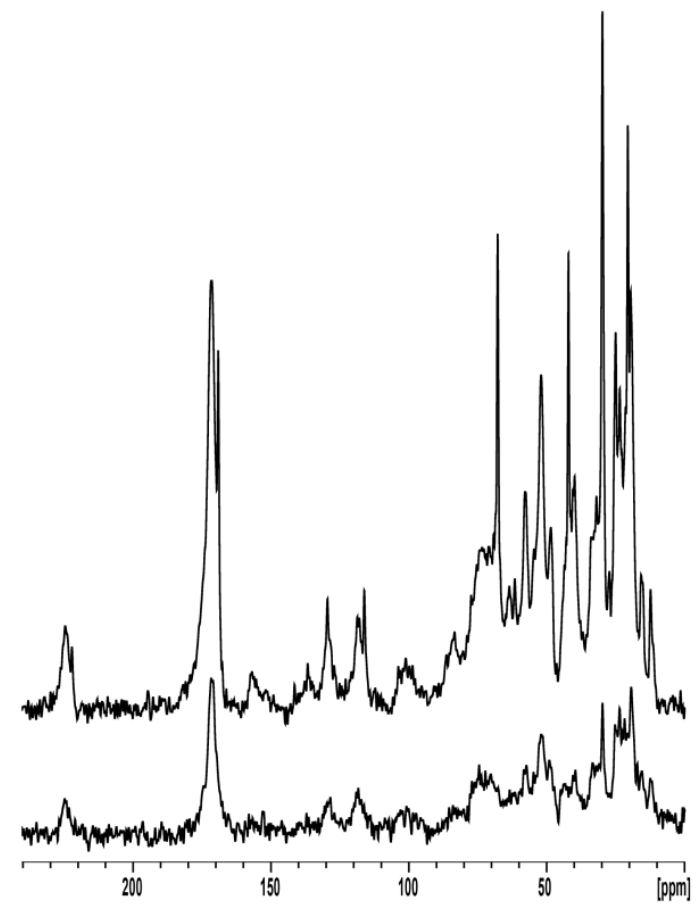

Figure S7. ${ }^{13} \mathrm{C}$ CP-MAS NMR spectra of BMMT (C) at different loading of biomass in (A), $\mathrm{D}_{2} \mathrm{O}$ and (B), DMSO- $\mathrm{d}_{6}$. 

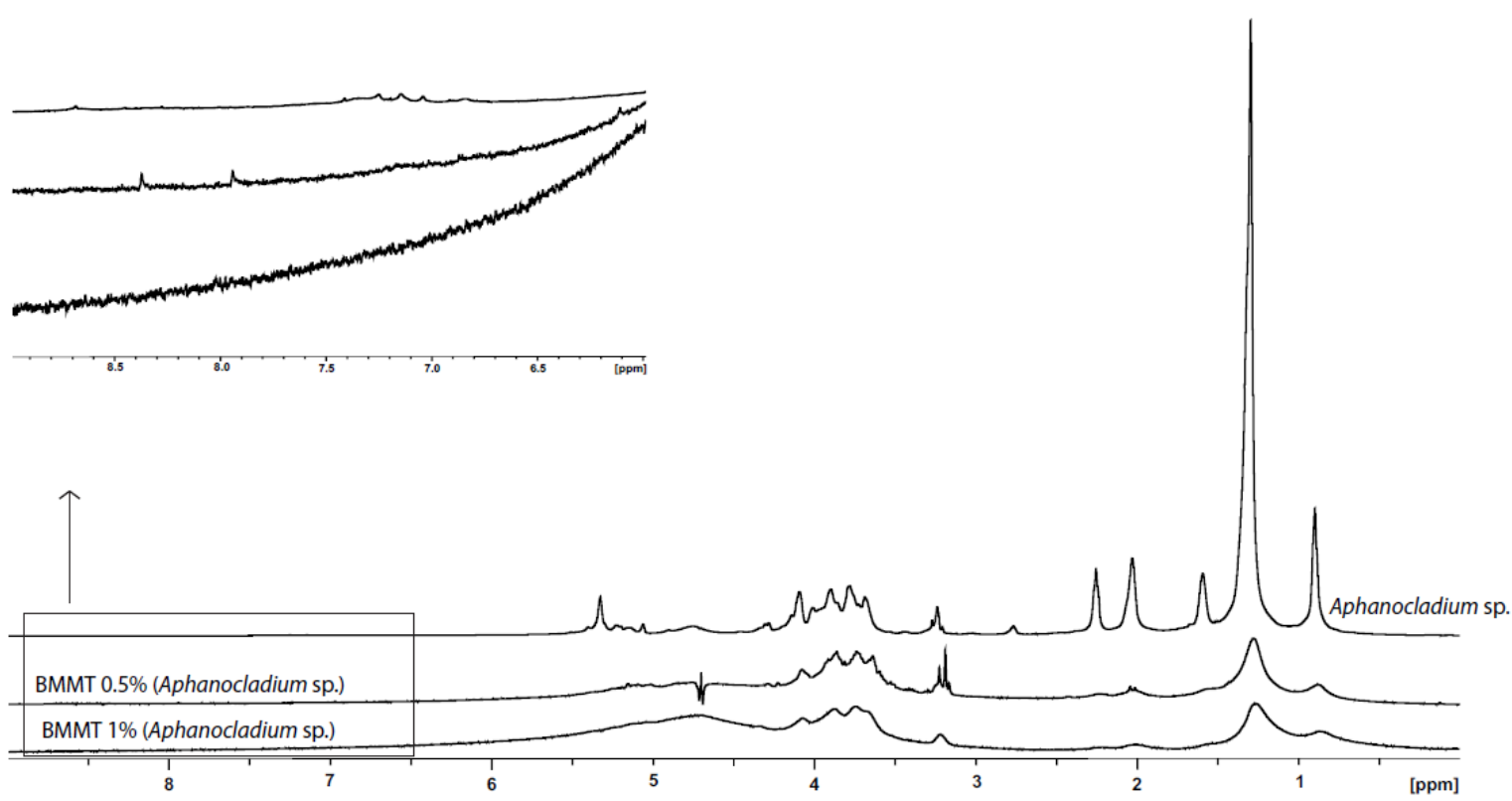

Figure S8. ${ }^{1} \mathrm{H}$ CMP-MAS NMR spectra of BMMT (Apha) at different loading of biomass in $\mathrm{D}_{2} \mathrm{O}$. Assignments are as follows: terminal methyl and methylene groups between 0.61.3 ppm; signals due to $O$ - and $N$ - substituted aliphatic structures in the 1.3-2.9 ppm region; signals due to $O$-alkyl structures mainly from carbohydrates in the 2.9-4.1 ppm region; overlapping weak resonances in the 4.1-5.4 ppm region generally attributed to a number of moieties including $\alpha-\mathrm{H}$ from peptides, anomeric $\mathrm{H}$ in carbohydrates and double bonds; and (box and arrow) signals from the aromatic structures, in the 6.2-8.5 ppm region.

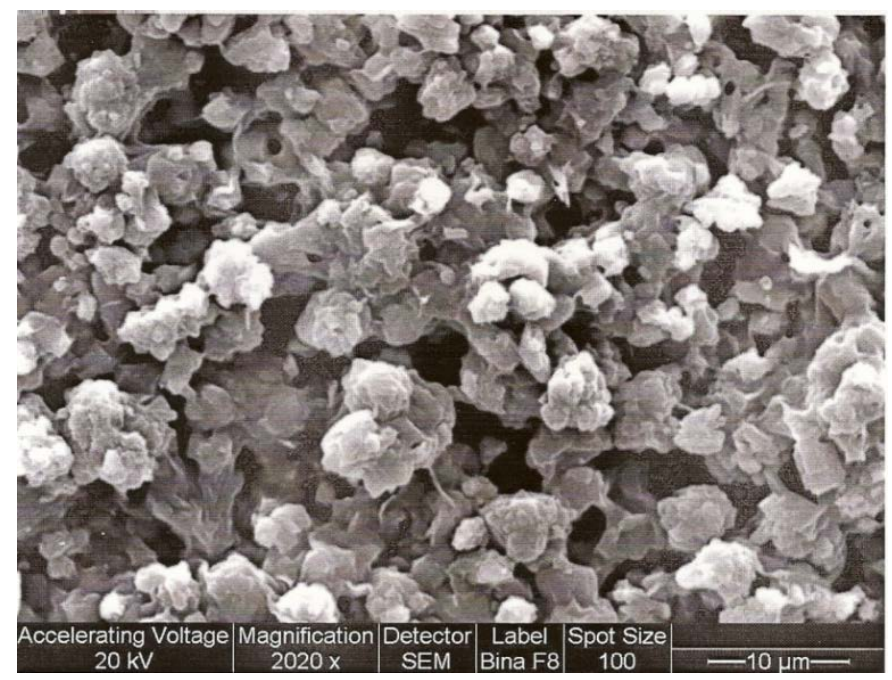

Figure S9. Scanning electron micrograph of MMT. 